\title{
HIVAN and medication use in chronic dialysis patients in the United States: analysis of the USRDS DMMS Wave 2 study Kevin C Abbott*1, Fernando C Trespalacios ${ }^{2}$, Lawrence Y Agodoa ${ }^{3}$ and Tejinder S Ahuja ${ }^{4}$
}

Address: ${ }^{1}$ Nephrology Service, Walter Reed Army Medical Center, Washington, DC, and Uniformed Services University of the Health Sciences, Bethesda, MD, USA, ${ }^{2}$ Nephrology Service, Madigan Army Medical Center, Tacoma, Washington, D.C, USA, ${ }^{3}$ NIDDK, NIH, Bethesda, MD, USA and ${ }^{4}$ Department of Medicine, Division of Nephrology, University of Texas Medical Branch, Galveston, TX, USA

Email: Kevin C Abbott* - Kevin.Abbott@NA.AMEDD.ARMY.MIL; Fernando C Trespalacios - Kevin.Abbott@NA.AMEDD.ARMY.MIL; Lawrence Y Agodoa - agodoal@extra.niddk.nih.gov; Tejinder S Ahuja - tahuja@utmb.edu

* Corresponding author

Published: 01 July 2003

BMC Nephrology 2003, 4:5

This article is available from: http://www.biomedcentral.com/I47/-2369/4/5

(c) 2003 Abbott et al; licensee BioMed Central Ltd. This is an Open Access article: verbatim copying and redistribution of this article are permitted in all media for any purpose, provided this notice is preserved along with the article's original URL.
Received: 25 March 2003

Accepted: 0I July 2003

\begin{abstract}
Background: The use and possible effects of factors known to improve outcomes in patients with human immunodeficiency virus associated nephropathy (HIVAN), namely of angiotensin converting enzyme inhibitors (ACE) and antiretroviral therapy, has not been reported for a national sample of dialysis patients.

Methods: We conducted a historical cohort study of the United States Renal Data System (USRDS) Dialysis Morbidity and Mortality Study (DMMS) Wave 2 to identify risk factors associated with increased mortality in these patients. Data were available for 3374 patients who started dialysis and were followed until March 2000. Cox Regression analysis was used to model adjusted hazard ratios (AHR) with HIVAN as a cause of end stage renal disease (ESRD) and its impact on mortality during the study period, adjusted for potential confounders.
\end{abstract}

Results: Of the 3374 patients who started dialysis, 36 (I.I\%) had ESRD as a result of HIVAN. Only $22(61 \%)$ of patients with HIVAN received antiretroviral agents, and only nine patients $(25 \%)$ received combination antiretroviral therapy, and only $14 \%$ received ACE inhibitors. Neither the use of multiple antiretroviral drugs (AHR, $0.62,95 \% \mathrm{Cl}, 0.10,3.86, p=0.60)$, or ACE inhibitors were associated with a survival advantage. Patients with HIVAN had an increased risk of mortality (adjusted hazard ratio, 4.74, 95\% Confidence Interval, 3.12, 7.32, p < 0.0I) compared to patients with other causes of ESRD.

Conclusions: Medications known to improve outcomes in HIV infected patients were underutilized in patients with HIVAN. Adjusted for other factors, a primary diagnosis of HIVAN was associated with increased mortality compared with other causes of ESRD.

\section{Background}

Previously limited to case series, [1-4] information on the patient characteristics and course of human immunodeficiency virus or acquired immunodeficiency syndrome 
associated nephropathy (HIVAN) after the onset of end stage renal disease (ESRD) has been reported for the national population of US ESRD patients. [5,6] Substantial improvements in the survival of dialysis patients with HIV infection have been noted after 1995,[6] and have been attributed to treatment with highly active antiretroviral therapy (HAART). [7] Despite these encouraging reports, the morbidity and mortality of these patients remains high compared with age-matched patients with ESRD from other causes. [6] Despite the improving information on the pharmacokinetics of these drugs in dialysis patients, a recent report suggested that only $58 \%$ of the 62 HIV-infected patients with ESRD were on antiretroviral therapy. [8] Use of angiotensin converting enzyme inhibitors (ACE) in patients with HIVAN is associated with delayed progression to ESRD. $[9,10]$ Some other reports have suggested that use of ACE in dialysis patients may improve survival. [11] It is possible the use of ACE might be particularly beneficial in HIV infected patients with ESRD particularly those with HIV associated nephropathy as heavy proteinuria often continues after the onset of dialysis [12]. Alternatively, risk of hyperkalemia from ACE in patients with HIVAN due to associated tubulointerstitial inflammation and renal tubular acidosis may increase mortality. [13] Although a recent single-center report found no benefit of ACE in dialysis patients with HIVAN [14], it is not clear whether this represents national experience.

Recent anecdotal reports of remission of HIVAN with use of HAART are encouraging. However, the prevalence of patients with HIVAN who develop ESRD may not decrease, because of improvement in survival of these patients, allowing them to live long enough to reach ESRD. [15-17] Such patients may even be more difficult to manage, since the onset of HIVAN could be delayed until these patients develop resistance to the therapy. Therefore, HIVAN is likely to continue as a major cause of ESRD in young African-Americans. Better understanding of factors associated with poor outcomes in patients with HIVAN and ESRD would help in improving outcomes in these patients. More detailed information on the metabolic, cardiovascular and chronic kidney disease specific characteristics of HIVAN patients on dialysis, as well as the use and possible benefit of certain medications, especially ACE and antiretroviral drugs in this population, would supplement previous reports. Further, use of a national database would allow the comparison of HIVAN patients in a large at-risk population and allow comparison with single center studies that may reflect practices that may not be representative of the national as a whole. Therefore, we analyzed data from the standard analysis files of the 2000 United States Renal Data System (USRDS) Dialysis Morbidity and Mortality (DMMS) Wave 2 database. The primary objective of the study was to examine whether patients with HIVAN who present to chronic dialysis had important differences in clinical and laboratory parameters compared to patients with other causes of ESRD and to assess factors associated with better survival such as laboratory parameters and medication use.

\section{Methods}

A historical cohort study of the USRDS DMMS Wave 2 was performed. Details on the inception, limitations, validity, variables and questionnaires used in the study are available online at the USRDS researcher's guide website, http:/ /www.usrds.org/research.htm. This database has been used in many previous cross-sectional and longitudinal studies including one by our own institution. [18]. Briefly, DMMS WAVE 2 was a prospective cohort study of a random sample of $20 \%$ of all U.S. hemodialysis patients and virtually all peritoneal dialysis patients starting treatment in 1996 and early 1997. Because patients who started dialysis in 1997 comprised only a small proportion of the study population, we limited analysis to patients who started dialysis in 1996. Also, because outcomes such as dates of death and follow-up had to be merged with this study from other USRDS files, we did not consider it a true prospective cohort study for the purposes of this analysis.

Characteristics of hemodialysis and peritoneal dialysis patients (abstracted from prospective surveys conducted specifically for DMMS WAVE 2) were matched and weighted to allow more appropriate comparisons between modalities. Baseline and follow-up data used in the study are shown in Table 1 [see Additional file: 1]. All results are reported as $\mathrm{N}(\%)$ or the mean \pm the standard deviation. Patients with ESRD due to HIVAN were determined from the variable PDIS (primary cause of renal failure) from the file SAF.PATIENTS and merged with the DMMS WAVE 2 files using unique patient identifiers. Causes of renal failure selected as HIVAN were 0429A, $0429 Z, 0439 Z$, or $0449 Z$ (various codes for HIV or AIDS associated nephropathy), from the variable PDIS in the file SAF.PATIENTS, Core CD and were coded as 1 in statistical analysis, with all other patients coded as 0. AIDS associated nephropathy was listed as a diagnosis in the years studied and therefore that term is still used in the present study. In addition, a maximum of 15 medications prescribed to each patient at the study start date (day 60 of dialysis) were recorded. From this list, the use of betablockers (both cardioselective and non-selective), angiotensin-converting enzyme (ACE) inhibitors, calcium channel blockers (subcategorized as dihyropyridine and non-dihydropyridine), 3-hydroxy-3-methylglutaryl coenzyme A (HMG-CoA) reductase inhibitors (statins), and aspirin was determined. Carvedilol, which has been independently associated with improved survival in certain populations, was approved for use by the FDA in February 1997, and was therefore not assessed. Blood pressure 
levels, systolic and diastolic, were obtained as the mean of three readings before and after dialysis, respectively. Of the study cohort, $2198(65.1 \%)$ of patients with the variable HIV and 2164 (64.1\%) with the variable AIDS, respectively, were either missing, unknown, or coded as "cannot disclose," and these variables were therefore not used in analysis.

Survival status was linked to the DMMS Wave 2 data from the 2000 USRDS Patients Standard Analysis File (SAF.PATIENTS) via unique patient identifiers assigned by the USRDS. The date and cause of death listed in a patient's SAF was obtained from a form submitted to the USRDS by the patient's nephrologist (form HCFA 2746). Descriptions of these files are available under the "USRDS Researcher's Guide" at http://www.usrds.org/ research.htm. Patient survival status was complete through March 2000. Survival time was defined as the time from 90 days after the date of the first dialysis session until the date of death, censored for receipt of renal transplant, loss to followup or the end of the study period. For comparison, models that did not censor for the date of receipt of renal transplant were also used.

\section{Statistical analysis}

Continuous variables are presented as mean \pm standard deviation unless otherwise specified. Univariate analysis was performed with Chi-Square testing for categorical variables (Fisher exact test used for violations of Cochran's assumptions, meaning fewer than 5 cases per cell) and student's t-test for continuous variables (Mann Whitney test or logarithmic transformation was used for variables without a Gaussian distribution, verified by visual inspection and goodness of fit tests (Chi Square for linear trend). Variables with borderline significance ( $\mathrm{p}$ value $<0.1$ in univariate analysis) and those thought likely to have a clinical relationship with HIVAN were entered into the multivariate models. Stepwise (forward step likelihood ratio method) Cox proportional hazards analysis was used to assess the association between baseline factors and HIVAN, independent of other factors. This method used the most computationally intensive method for calculating mean hazard ratios (approximations of relative risk) entering each variable into the model and removing those that were no longer significant after adjustment for all other variables. Log-log plots were inspected to verify the existence of proportional hazards. Estimated hazard ratios (HR) along with corresponding 95\% confidence intervals (CI) and p values are reported for all regression covariates. The association of HIVAN with mortality was also tested by Cox regression analysis using adjusted hazard ratios (AHR). Hierarchically well-formed models were used in the assessment of interaction terms. Collinearity diagnostics were evaluated both from the output of the statistical procedures and by manual generation of mod- els including only one of several variables with close relationships (for example, malnutrition, body mass index and serum albumin). To assess for possible confounding, logistic regression was also performed to assess for significant associations with a history of HIVAN using the same covariates as for Cox Regression as above. Multiple methods were used to assess goodness of fit with all variables, including Nagelkerke $\mathrm{r}^{2}$ (an attempt to quantify the proportion of explained "variation" in the logistic regression model, although values for $\mathrm{r}^{2}$ in logistic regression are much lower than for linear regression) and the c-statistic, which is the receiver operating curve for the cumulative predicted probability of the logistic regression model $(0.5$ being equivalent to chance and 1.0 equivalent to $100 \%$ agreement).

\section{Results}

A total of 4065 patients were included in the DMMS Wave 2 cohort. Of these, 3621 patients had valid dates for starting dialysis in 1996. From this cohort, 3374 had sufficient information to calculate follow-up times. Thirty-six of these patients had ESRD as a result of HIVAN. Mean follow-up was $2.19 \pm 1.14$ (standard deviation) years. Characteristics of all the patients and patients with HIVAN are summarized in Table 1 (see Additional file 1). As shown, in this file factors positively associated with HIV in univariate analysis included male gender, African American race, younger age, lower body mass index, decreased comorbidity in general, increased rates of malnutrition and smoking, lower blood pressure and cholesterol, higher serum creatinine, lower serum albumin bicarbonate, and decreased use of aspirin and calcium channel blockers. The distribution of serum PTH levels in the study population was severely skewed, with a skewness of 2.49 and a kurtosis of 9.08. We elected to use non-parametric tests of association (the Mann-Whitney test) and a t-test of logarithmically transformed values of PTH. Although the difference in serum PTH levels between patients with HIVAN and patients with other causes of renal disease appeared large, it was not significant in Mann-Whitney testing ( $\mathrm{p}=$ 0.34 ) or t-tests of logarithmically transformed values of serum PTH $(p=0.64)$.

Specific antiretroviral agents are also shown in this file. The most common agents used in HIVAN patients were zidovudine and lamivudine. Only $22(61 \%)$ of patients with HIVAN received antiretroviral agents, and only nine patients $(25 \%)$ received combination antiretroviral therapy. Five patients received both zidovudine and lamivudine (a combination later called Combivir ${ }^{\mathrm{TM}}$ ). Other combinations included one each for zidovudine-zalcitabine, stavudine-saquinavir, stavidine-lamivudine, and indinavir-lamivudine. 
Table 2: Logistic Regression of Factors Associated with HIVAN, entire cohort

\begin{tabular}{|c|c|c|c|}
\hline & $P$ value & Adjusted odds ratio for HIVAN & $95 \% \mathrm{Cl}$ \\
\hline Age $<48$ (vs. $>=71$ )* & 0.005 & 9.35 & $1.99,43.48$ \\
\hline Serum albumin (per higher quartile) & $<0.001$ & 0.31 & $0.15,0.69$ \\
\hline Male (vs. female) & 0.001 & 6.58 & $2.39,18.11$ \\
\hline African American & $<0.001$ & 11.03 & $4.09,27.78$ \\
\hline CCB use (vs. other medications) & 0.02 & 0.23 & $0.07,0.75$ \\
\hline Malnourished (vs. adequate nutrition) & 0.001 & 4.09 & $1.56,10.72$ \\
\hline Smoking (vs. non active smoking) & 0.02 & 2.89 & $1.16,7.18$ \\
\hline
\end{tabular}

HIVAN=Human immunodeficiency virus associated nephropathy. *Other age categories were not statistically significant when compared to age $>=$ $7 \mathrm{I}$ years Nagelkerke ${ }^{2}$ was 0.50 , c-statistic was $0.97(95 \% \mathrm{Cl}, 0.96,0.99)$

Table 1 shows factors independently associated with HIVAN in logistic regression. Factors included antiretroviral therapy, male gender, African American race, younger age, lower serum albumin, higher rates of malnutrition and smoking, and lower use of calcium channel blockers. There were no significant differences in ACE use by either HIV or HIVAN status, either unadjusted or adjusted. There was no significant interaction between ACE and antiretroviral therapy.

Patients with HIVAN had significantly lower survival compared to patients with other causes of ESRD (Table 2, Figure 1). Plots of survival by ACE use are shown for HIVAN patients (Figure 2) and for the entire study cohort (Figure 3). In Cox Regression analysis adjusted for other factors known to be associated with mortality, HIVAN was independently associated with increased risk of mortality, adjusted hazard ratio, 4.74, 95\% CI, 3.12-7.32, p < 0.0001 . ACE use was not significantly associated with mortality in the entire cohort in Cox Regression, and it was not significant as an interaction term with HIVAN (p $=0.38$, adjusted hazard ratio, $1.64,95 \%$ CI, 0.55-4.87). In stratified analysis limited to patients with HIVAN, ACE use was not significantly associated with mortality ( $\mathrm{p}=$ $0.48, \mathrm{AHR}=1.88,95 \% \mathrm{CI}, 0.33-10.83$ ). Although the risk of death of patients with HIVAN and ESRD treated with single antiretroviral drug was higher than those not taking antiretroviral agents, patients on multiple antiretroviral drugs had a lower hazard ratio in adjusted analysis $(0.62$, 95\% CI, $0.10-3.86$ by Cox Regression), however this was not statistically significant (Figure 4). Among patients with HIVAN, the leading causes of death were AIDS $(50 \%)$, missing/unknown $(10.7 \%)$, and cardiac arrest of unknown cause $(10.7 \%)$. Factors independently associated with survival in HIVAN patients are shown in Table 3. The only factor independently associate with improved survival was African American race, compared to all other races.

\section{Discussion}

The present study found that only $61 \%$ of a national sample of US dialysis patients with HIVAN received antiretroviral therapy, consistent with previous regional reports of $58 \%$ use of anti-retroviral agents, [8] suggesting that underutilization of antiretroviral in dialysis patients with HIVAN is a national problem. HAART use, which the present study could not directly measure, is also low in dialysis patients with HIVAN, at $33 \%$ according to a recent single-center study [14]. We found no beneficial effect of ACE on survival in HIVAN, also consistent with previous studies [14]. The survival of HIVAN patients on dialysis in the preset study is also consistent with previous reports $[5,6]$. One-year survival of patients with HIVAN was only $53 \%$ compared to $83 \%$ for patients with all other causes of ESRD. This survival is lower than reported recently by Ahuja et al. [6] in HIV infected patients starting dialysis in the United States after 1997, likely from the fact that patients included in the DMMS wave 2 were recruited in 1996. Although data on CD4 counts and viral loads were unavailable from the database (the importance of which is illustrated in two reviews.[19,20] HIVAN is generally a late manifestation of HIV infection. [21] Therefore, use of antiretroviral therapy would be expected in patients with HIVAN in the absence of definite contraindications, even disregarding the previously cited reports of a possible beneficial effect of HAART on the course of HIVAN itself. However, in the present study only $61 \%$ of patients with HIVAN were receiving antiretroviral therapy and $41 \%$ of these were only on single antiretroviral therapy. Although not statistically significant, there was a trend towards better survival of patients on two antiretroviral drugs. None of the patients was on more then two antiretroviral drugs. This underutilization of antiretroviral therapy could be due hesitancy on the part of the infectious disease specialists and nephrologists for using these drugs due to unavailability of data on the pharmacokinetics of these drugs in patients on dialysis. Providers may also be concerned about the nephrotoxicity of certain antiretroviral agents, notably indinavir-associated crystal induced renal failure, [22] and other antiretroviral agents that require dose 


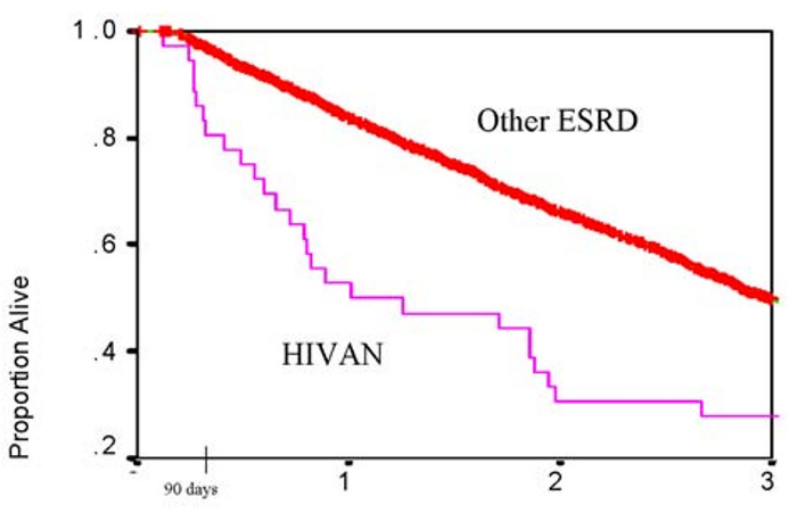

Figure I

Survival, Dialysis Morbidity and Mortality Wave II Cohort (DMMS II), 1996 only, N = 3374, patients with Human Immunodeficiency Virus (HIV) associated nephropathy (HIVAN) vs. patients with other causes of end stage renal disease (ESRD), $\mathrm{p}<0.0$ I by Log Rank Test.

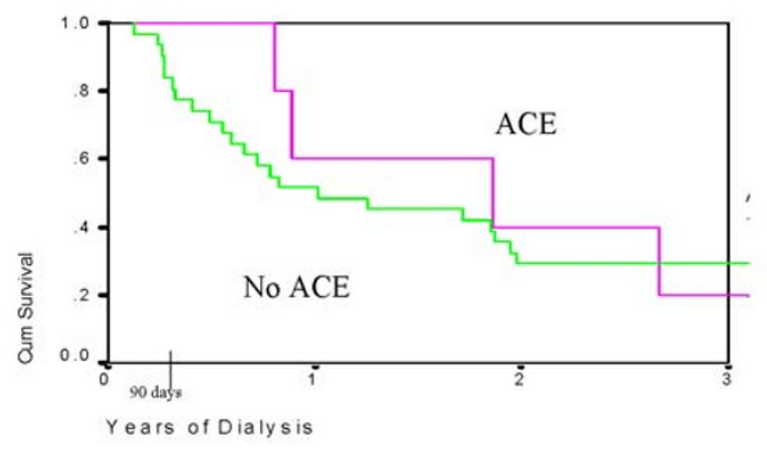

\section{Figure 2}

Survival in HIVAN infected patients on chronic dialysis, 1996 DMMS II, by angiotensin converting enzyme inhibitor (ACE) use. $P=0.53$ by Log Rank Test.

adjustment in renal failure. [22] Urgent studies are therefore needed to understand factors responsible for this underutilization of HAART in these patients. Similar underutilization of medications known to be beneficial in patients with chronic kidney disease has also been reported for cardiovascular disease. [18,23], suggesting such practice may not be an isolated phenomenon. How-

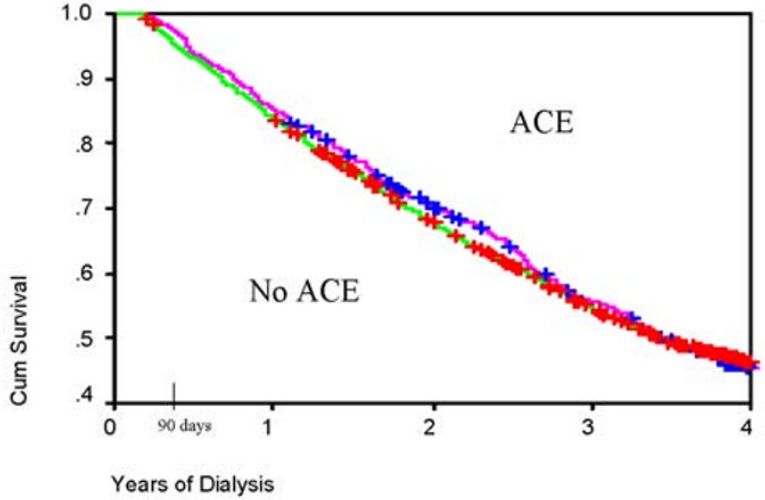

Figure 3

Survival in patients on chronic dialysis, 1996 DMMS II, by angiotensin converting enzyme inhibitor (ACE) use. $P=0.85$ by Log Rank Test.

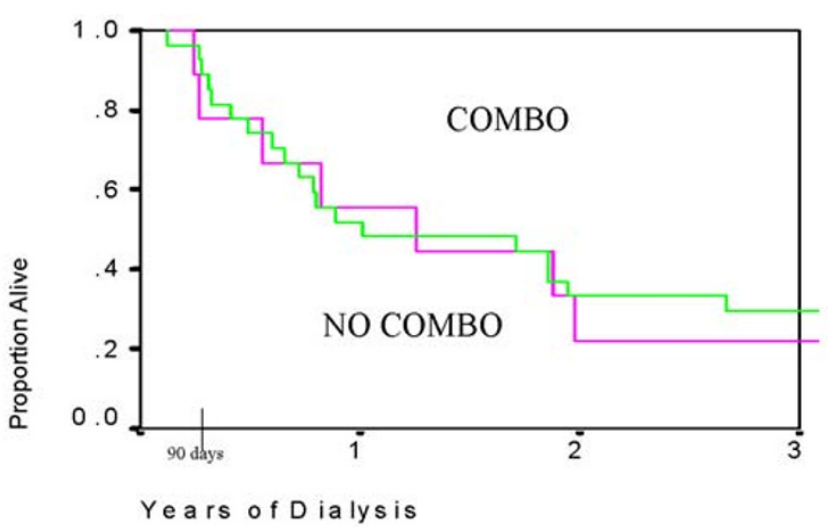

Figure 4

Survival, Dialysis Morbidity and Mortality Wave II Cohort (DMMS II), 1996 only, N = 3374, patients with Human Immunodeficiency Virus (HIV) associated nephropathy (HIVAN) who received combination retroviral therapy (COMBO) vs. all those who did not (NO COMBO), including those who were treated with single anti-retroviral agents, $P=0.77$ by Log Rank Test.

ever, the long term tolerability and efficacy of HAART has not been studied in patients on dialysis.

Although a benefit of ACE use in HIV infected patients has been reported previously, [9] the benefits of ACE in retarding progression of renal failure in patients with HIVAN did not translate into improvement of survival of these patients on dialysis. This is in agreement with some 
Table 3:

\begin{tabular}{|c|c|c|c|}
\hline Percent survival & I-year & 2-year & 3-year \\
\hline HIVANA & 53 & 31 & 28 \\
\hline Patients at risk & 19 & II & 10 \\
\hline Dialysis Patients with other causes of renal failure & 83 & 66 & 49 \\
\hline Patients at risk & 2707 & 2180 & 1722 \\
\hline
\end{tabular}

AUnadjusted cumulative percent survival, censored for receipt of renal transplant HIVAN=ESRD due to HIV/AIDS associated nephropathy AIDS=acquired immunodeficiency syndrome

Table 4: Cox Regression of Factors Associated with Mortality, DMMS WAVE 2 patients with HIVAN only

\begin{tabular}{|c|c|c|c|c|c|}
\hline & $\begin{array}{l}\text { One-Year Unadjusted } \\
\text { Survival Factor Present }\end{array}$ & $\begin{array}{l}\text { One-Year Unadjusted } \\
\text { Survival Factor Absent }\end{array}$ & P Value & AHR & $95 \% \mathrm{Cl}$ \\
\hline \multirow[t]{2}{*}{ African American Race* (vs. all other races) } & $54 \%$ & $40 \%$ & 0.05 & 0.20 & $0.04,0.99$ \\
\hline & Two-Year Unadjusted Survival & Two-Year Unadjusted Survival & & & \\
\hline Antiretroviral therapy (yes/No) & $15 \%$ & $39 \%$ & 0.21 & 1.92 & $0.69,5.37$ \\
\hline Multiple antiretroviral agents (yes/No) & $22 \%$ & $33 \%$ & 0.60 & 0.62 & $0.10,3.86$ \\
\hline ACE inhibitors (vs. nonuse) & $13 \%$ & $29 \%$ & 0.28 & 2.26 & $0.55,9.31$ \\
\hline CCB (vs. nonuse) & $42 \%$ & $25 \%$ & 0.36 & 0.56 & $0.16,1.96$ \\
\hline Male (vs. female) & $33 \%$ & $17 \%$ & 0.31 & 0.44 & $0.09,2.16$ \\
\hline Peritoneal Dialysis (vs. Hemodialysis) & $38 \%$ & $26 \%$ & 0.47 & 0.58 & $0.13,2.53$ \\
\hline
\end{tabular}

$\mathrm{N}=36, \mathrm{~N}$ in final model $=30 *$ Because so few patients with HIVAN were not African American, only one-year survival could be compared Only factors significant in the final model are shown.

recommendations that ACE use, to be effective, must start early in the course of HIVAN. As the number of patients with HIVAN who were treated with ACE was small in the present cohort, further prospective studies are required to validate our initial observation.

In comparison to patients with other causes of ESRD, the patients with HIVAN included in the DMMS wave 2 study were younger, the majority were African-Americans and had lower serum albumin and more severe acidosis. Some reports have suggested that HIV can infect parathyroid cells and these patients have low parathyroid hormone (PTH) and 1,25 dihydroxyvitamin D3 levels. [24] However, this did not prevent development of secondary hyperparathyroidism in patients with HIVAN and ESRD. The mean PTH level of the patients with HIVAN was 225 $\pm 42.6 \mathrm{pcg} / \mathrm{L}$, although lower was not statistically different from patients with causes of ESRD.

The present study has several limitations: it is observational and not randomized, therefore, confounding might persist despite adjustment. It is a random sample of all hemodialysis patients, not the total population, although the peritoneal dialysis population is almost fully captured. Information bias could have arisen due to mistakes in coding, especially for medications. We did not know the duration of use of medications prior to the study start. We were also unable to follow changes in variables over time. Therefore, we could not follow changes in blood pressure, laboratory values, or dialysis adequacy. This most especially applied to possible changes in medication use and changes in patient dry weight. These same limitations apply to other published studies using this database. In addition the information on CD4 count and plasma viral load in patients with HIVAN was not available and this could be an important uncorrected confounding factor. We could not assess factors associated with HIV seropositivity or AIDS due to the high percentage of patients with missing values for these variables.

In summary, we conclude that patients with HIVAN have a lower survival compared to patients with ESRD from other causes. Antiretroviral therapy is underutilized and the use of ACE does not improve survival of these patients. Future prospective studies are required to determine efficacy and tolerability of HAART and ACE inhibitors in patients with HIVAN and ESRD.

\section{Competing interests}

None declared. 


\section{Authors' contributions}

KCA performed the primary data analysis and collaborated in the writing of the manuscript.

FCT derived the algorithms for the medication variables and collaborated in the writing of the manuscript.

LYA provided the USRDS CD data files and supervised the project, and collaborated in the writing of the manuscript.

TSA collaborated in the writing of the manuscript.

\section{Note}

The opinions are solely those of the authors and do not represent an endorsement by the Department of Defense or the National Institutes of Health. This is a U.S. Government work. There are no restrictions on its use

\section{Additional material}

\section{Additional file 1}

Click here for file

[http://www.biomedcentral.com/content/supplementary/14712369-4-5-S1.doc]

\section{References}

I. al-Sulaiman M, al-Khader AA, al-Hasani MK and Dhar JM: Impact of HIV infection on dialysis and renal transplantation Transplant Proc 1989, 21: | 970-1971.

2. Feinfeld DA, Kaplan R, Dressler R and Lynn RI: Survival of human immunodeficiency virus-infected patients on maintenance dialysis Clin Nephrol 1989, 32:22I-224.

3. Obayomi OO, Dillard MG, Hosten AO and Cruz IA: End-stage renal disease in patients infected with human immunodeficiency virus: a retrospective review of $\mathbf{3 8}$ cases J Natl Med Assoc 1995, 87:762-765.

4. Perinbasekar S, Brod-Miller C, Pal S and Mattana J: Predictors of survival in HIV-infected patients on hemodialysis Am J Nephrol 1996, 16:280-286.

5. Abbott KC, Hypolite I, Welch PG and Agodoa LY: Human immunodeficiency virus/acquired immunodeficiency syndromeassociated nephropathy at end-stage renal disease in the United States: patient characteristics and survival in the pre highly active antiretroviral therapy era J Nephrol 200I, I 4:377383.

6. Ahuja TS, Grady J and Khan S: Changing trends in the survival of dialysis patients with human immunodeficiency virus in the United States J Am Soc Nephrol 2002, 13:1889-1893.

7. Ahuja TS, Borucki M and Grady J: Highly active antiretroviral therapy improves survival of HIV-infected hemodialysis patients Am J Kidney Dis 2000, 36:574-580.

8. Szczech LA, Kalayjian R, Rodriguez R, Gupta S, Coladonato J and Winston J: The clinical characteristics and antiretroviral dosing patterns of HIV-infected patients receiving dialysis Kidney Int 2003, 63:2295-230I.

9. Kimmel PL, Mishkin GJ and Umana WO: Captopril and renal survival in patients with human immunodeficiency virus nephropathy Am J Kidney Dis 1996, 28:202-208.

10. Burns GC, Paul SK, Toth IR and Sivak SL: Effect of angiotensinconverting enzyme inhibition in HIV-associated nephropathy J Am Soc Nephrol 1997, 8: I I 40-I I 46.
II. Efrati S, Zaidenstein R, Dishy V, Beberashvili I, Sharist M, Averbukh Z, Golik $A$ and Weissgarten J: ACE inhibitors and survival of hemodialysis patients Am J Kidney Dis 2002, 40: 1023-9.

12. Laradi A, Mallet A, Beaufils H, Allouache M and Martinez F: HIVassociated nephropathy: outcome and prognosis factors. Groupe d' Etudes Nephrologiques d'lle de France J Am Soc Nephrol 1998, 9:2327-35.

13. Sheehan MT and Wen SF: Hyperkalemic renal tubular acidosis induced by trimethoprim/sulfamethoxazole in an AIDS patient Clin Nephrol 1998, 50:I88-93.

14. Rodriguez RA, Mendelson M, O'Hare AM, Hsu LC and Schoenfeld P: Determinants of Survival among HIV-Infected Chronic Dialysis Patients J Am Soc Nephrol 2003, 14:1307-13.

15. Ross MJ and Klotman PE: Recent Progress in HIV-Associated Nephropathy J Am Soc Nephrol 2002, 13:2997-3004.

16. Winston JA, Bruggeman LA and Ross MD et al:: Nephropathy and establishment of a renal reservoir of HIV type I during primary infection N Engl J Med 200 I, 344: 1979-1984.

17. Kirschner JT: Resolution of renal failure after initiation of HAART: 3 cases and a discussion of the literature AIDS Read 2002, 1 2: 103-105.

18. Trespalacios FC, Taylor AJ, Agodoa LY and Abbott KC: Incident acute coronary syndromes in chronic dialysis patients in the United States Kidney Int 2002, 62:1799-1805.

19. Weller IV and Williams IG: ABC of AIDS. Antiretroviral drugs $B M J$ 322(7299): 14I0-2. 200I Jun 9

20. Mindel $A$ and Tenant-Flowers M: ABC of AIDS: Natural history and management of early HIV infection $B M]$ 322(7297): 1290 3. 2001 may 26

21. Rao TK: Human immunodeficiency virus infection and renal failure Infect Dis Clin North Am 200 I, I 5:833-850.

22. Reilly RF, Tray K and Perazella MA: Indinavir nephropathy revisited: a pattern of insidious renal failure with identifiable risk factors Am J Kidney Dis 200I, 38:E23.

23. Shlipak MG, Heidenreich PA, Noguchi H, Chertow GM, Browner WS and McClellan MB: Association of renal insufficiency with treatment and outcomes after myocardial infarction in elderly patients Ann Intern Med 2002, I37:555-562.

24. Haug CJ, Aukrust P, Haug E, Morkid L, Muller F and Froland SS: Severe deficiency of 1,25-dihydroxyvitamin D3 in human immunodeficiency virus infection: association with immunological hyperactivity and only minor changes in calcium homeostasis J Clin Endocr Metab 1998, 83:383-388.

\section{Pre-publication history}

The pre-publication history for this paper can be accessed here:

http://www.biomedcentral.com/1471-2369/4/5/prepub

Publish with Bio Med Central and every scientist can read your work free of charge

"BioMed Central will be the most significant development for disseminating the results of biomedical research in our lifetime. "

Sir Paul Nurse, Cancer Research UK

Your research papers will be:

- available free of charge to the entire biomedical community

- peer reviewed and published immediately upon acceptance

- cited in PubMed and archived on PubMed Central

- yours - you keep the copyright 